\title{
Tamizaje del cáncer de cuello uterino con test de HVP. Primeros resultados en el sistema público de Uruguay
}

Guillermo Rodríguez*, Laura García†, Andrea Beracochea, Rafael Alonso§,

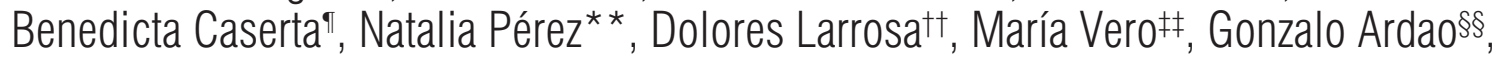
Mary Nela Santos" y grupo ESTAMPA Uruguay*

\section{Resumen}

Introduccción: en Uruguay, el cáncer de cuello uterino ocupó el tercer lugar con una incidencia promedio de 312 nuevos casos por año; fallecieron 134 mujeres promedio por año en el período 2010 - 2014.

Objetivo: el objetivo de este trabajo fue analizar los primeros resultados de una experiencia piloto con la aplicación del test de HPV captura híbrida HC2 (Qiagen ${ }^{R}$ ) como test de tamizaje primario del cáncer de cuello uterino en una zona del departamento de Canelones.

Método: se estudiaron 1.010 mujeres asintomáticas entre 30 y 64 años que concurrieron a realizarse el test de PAP a dos unidades de toma de muestras del Programa de prevención del cáncer de cuello uterino. Se realizó la extracción conjunta de la muestra para PAP, que fue derivada a uno de los laboratorios de citología de la Red de Atención Primaria de Salud y la muestra de HPV que fue procesada con la técnica de captura híbrida en el laboratorio de biología molecular del Centro Hospitalario Pereira Rossell. Las usuarias con resultados HPV + y PAP anormales (ASC-US+) fueron derivadas a colposcopia, con biopsia y posterior tratamiento cuando correspondió.

Resultados: el test de HPV fue positivo en 126/1.010 mujeres (12,5\%) y el PAP anormal en 167/1.010 (16,5\%). El test de

\footnotetext{
* Ex Prof. Adjunto de Clínica Ginecotocológica "C" Facultad de Medicina, UDELAR. Coordinador del Área de Prevención del Cáncer de Cuello Uterino de la Comisión Honoraria de Lucha contra el Cáncer.

† Prof. Adjunta Phd. Jefe de Laboratorio de Biología Molecular, Departamento de Patología Clínica, Centro Hospitalario Pereira Rossell - ASSE. ‡ Médico ginecólogo colposcopista, Centro de Salud de la Costa - ASSE.

$\S$ Prof. Titular del Departamento de Métodos Cuantitativos, Facultad de Medicina, UDELAR. Técnico asesor del Área de Prevención del cáncer de cuello uterino de la Comisión Honoraria de Lucha contra el Cáncer.

II Prof. Agregado de Cátedra de Anatomía Patológica, Facultad de Medicina, UDELAR. Jefe de Laboratorio de Anatomía Ptológica, Centro Hospitalario Pereira Rossell - ASSE.

** Prof. Adjunta de Clínica Ginecotocológica "B”. Facultad de Medicina, UDELAR.

†† Asistente social, Área de Prevención del cáncer de cuello uterino de la Comisión Honoraria de Lucha contra el Cáncer.

拉 Médico citólogo, Laboratorio RAP, RIEPS - ASSE.

$\S \S$ Médico patólogo. Jefe del Banco de Tumores de SSFFAA.

Tा Médico ginecólogo colposcopista, Centro de Salud de Pando - ASSE.

${ }_{* * *}$ Scientist, International Agency for Research on Cancer, World Health Organization.

††† Implementation Scientist. Head, Prevention and Implementation Group. International Agency for Research on Cancer, World Health Organization

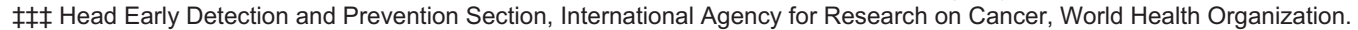

Grupo Estampa Uruguay: Dra. Elena González, Téc. Samanta Amigo, Dra. Adela Sica, Téc. Rosario Nesti, Téc. Romina Gabrielli, Téc. Claudia Carrera, Téc. Maguela Sosa, Dra. Adriana Della Valle, Dra. Nora Artagaveytia, Dra. Florencia Neffa, Lic. Marta Sapone, Florencia Carusso, Carolina Vergara, Melina Yorio, As. Beatriz Rivero, Rossana Gorini, Dra. María Gabriela Ilarramendi, Part. Marta Arcieri

Los autores declaran no tener conflicto de intereses

Maribel Almonte y Rolando Herrero son funcionarios de la Organización Mundial de la Salud. Las opiniones expresadas en la presente publicación son responsabilidad exclusiva de los autores, y no representan necesariamente las decisiones, el criterio ni la política de la Organización Mundial de la Salud.

Recibido: 3/4/19

Aprobado: $14 / 8 / 19$
} 
HPV fue positivo en todos los casos CIN2+ $13 / 13(100 \%)$ mientras que el PAP fue anormal (ASCUS+) en 7/13 (54\%) para CIN2+ por biopsia.

Conclusión: el test de HPV resultó más eficaz y eficiente que el PAP para la detección de lesiones precancerosas de cuello uterino.

Palabras clave: Neoplasias del cuello uterino

Infecciones por papillomavirus

Prueba de Papanicolaou

Cribado

Prevención secundaria

\author{
Key words: $\quad$ Uterine cervical neoplasms \\ Papillomavirus infections \\ Papanicolaou test \\ Screening \\ Secondary prevention
}

\section{Introducción}

El cáncer de cuello uterino es una de las enfermedades neoplásicas más frecuentes en las mujeres de los países de Latinoamérica. En el mundo fallecen anualmente más de 314.000 mujeres por esta causa y en Latinoamérica, $28.300^{(1)}$. En algunos países de Latinoamérica esta neoplasia ocupa los primeros lugares como causa de muerte más frecuente en las mujeres.

En Uruguay se diagnosticaron en promedio 312 nuevos casos por año y fallecieron 134 mujeres promedio por año por esta causa en el período 2010-2014 ${ }^{(2)}$. Ocupa en nuestro país el tercer lugar en incidencia como neoplasias más comunes de la mujer, luego del cáncer de mama y colorrecto.

Desde el inicio de la década de 1990 la Comisión Honoraria de Lucha contra el Cáncer (CHLCC) organizó un Programa de prevención del cáncer de cuello uterino (PPCCU) utilizando el test de Papanicolaou (PAP) convencional $^{(3)}$ como test de tamizaje primario. En los informes de Papanicolaou se utilizó la terminología Bethesda 2001. La población objetivo del programa fueron mujeres entre 30 y 64 años. La repetición del tamizaje fue cada tres años cuando el test fue negativo. Se derivaron para diagnóstico a colposcopía todas aquellas mujeres con resultado células escamosas de significado incierto (ASC-US) y mayor sospecha (ASC-US+). De esta forma, se creó una estructura a nivel nacional que aseguró el acceso al tamizaje mediante el PAP y el diagnóstico de las lesiones precancerosas por medio de la colposcopía.

Se alcanzó una cobertura de PAP a nivel nacional promedio de $44 \%$ en el período 1/10/2010 a 31/10/2013, en mujeres de 30 a 64 años con por lo menos un PAP en los últimos tres años (datos obtenidos de la memoria anual del PPCCU-CHLCC del año 2014).

Se ha verificado un cambio en la tendencia de la incidencia y la mortalidad por esta enfermedad en Uruguay.
Según el Registro Nacional de Cáncer de Uruguay se comprobó un descenso de $2,5 \%$ por año en el período 2002-2014 y una tasa de mortalidad estimada que descendió significativamente, $1,5 \%$ por año en el período 1998-2016. La tasa ajustada de incidencia del cáncer de cuello uterino es de 14,4 por 100.000 en el período 2010-2014 y la mortalidad tiene una tasa ajustada de 5,3 por 100.000 en el mismo período. Estos valores son los más bajos de los países de la región, pero muy superiores a los registrados en los países desarrollados, como es el caso de Finlandia, con una tasa de incidencia ajustada de 4,3 por 100.000 y una tasa de mortalidad ajustada de 1,0 por 100.000 para el año $2012^{(1)}$.

En setiembre del año 2012, el PPCCU pasó a depender del Ministerio de Salud (MS), sin mayores cambios en cuanto a la estructura de unidades de toma de muestras y laboratorios de citología ginecológica.

La población objetivo se extendió a mujeres de 21 a 69 años.

El cáncer de cuello uterino es una de las neoplasias que tiene una causa conocida e identificada que es la infección por papilomavirus humano (HPV) ${ }^{(4-6)}$. El HPV produce infecciones transitorias en la mayoría de los casos, sobre todo en mujeres menores de 30 años. En mujeres de 30 años y mayores también son frecuentes las infecciones transitorias, pero en menor proporción. En pocos casos se hacen persistentes, se introducen en el núcleo celular y producen su transformación. Esto lleva a la aparición de lesiones intraepiteliales escamosas de alto grado (H-SIL), neoplasia intraepitelial grado 2 y lesiones mayores (CIN 2+), que son los verdaderos precursores del cáncer de cuello uterino ${ }^{(7)}$. El tratamiento adecuado y oportuno de estas lesiones intraepiteliales constituye la forma efectiva de lograr la prevención secundaria del cáncer de cuello uterino. La identificación de lesiones intraepiteliales precursoras del cáncer se 
puede hacer empleando diversos métodos de tamizaje. El más antiguo y que ha demostrado en algunos países su eficacia en programas organizados $u$ oportunistas es el PAP, que consiste en la detección de anormalidades morfológicas sugestivas de lesiones relacionadas con el HPV. Para que la prevención secundaria sea efectiva es necesario lograr una alta cobertura $(80 \%)$ de la población objetivo y el seguimiento completo de las anormalidades detectadas. El PAP tiene la limitante de poseer una baja sensibilidad para detectar lesiones, por lo que debe repetirse con frecuencia para solventar parcialmente esa deficiencia, no siendo infrecuente la aparición de lesiones invasoras en mujeres con PAP normal.

La Organización Mundial de la Salud (OMS) recomienda a todos los países, como prevención primaria de la enfermedad, la incorporación de la vacunación profiláctica contra el virus del HPV de la población objetivo primaria, que son las niñas de 9 a 13 años, y la prevención secundaria mediante el tamizaje de las mujeres para diagnosticar lesiones precancerosas, logrando una alta cobertura y seguimiento, con algunos de los tests disponibles: PAP, test de HPV o inspección visual con ácido acético (IVAA) ${ }^{(8)}$.

En Uruguay se dispone en el subsector público del Sistema Nacional Integrado de Salud (SNIS) de 12 laboratorios de citología cérvico uterina, que han demostrado su eficacia en el tamizaje de la enfermedad. Pero no se dispone por el momento de citotécnicos como para aumentar la cobertura de la población de acuerdo con los requerimientos asistenciales. La mayoría de los informes de Papanicolaou, 70.000 al año, son realizados por médicos citopatólogos que están al límite de su dedicación para el tamizaje.

El test de HPV es una metodología nueva de tamizaje que ha demostrado tener una alta sensibilidad para la captación de lesiones precursoras del cáncer de cuello uterino, con otras ventajas adicionales, como son la posibilidad de que la mujer se tome su propia muestra (autotoma), la automatización de los procedimientos de laboratorio y la reproducibilidad de los diagnósticos. La alta sensibilidad y confiabilidad de la prueba permite extender los intervalos de tamizaje por varios años.

La prueba utilizada en esta investigación fue la captura de híbridos (HC2), desarrollada originalmente por DIGENE ${ }^{\mathrm{R}}$ Corporation (Estados Unidos) y actualmente comercializada por Qiagen $^{\mathrm{R}(9)}$.

Se trata de una de las pruebas más antiguas utilizadas en el tamizaje y la más empleada por los diferentes programas de prevención del cáncer de cuello uterino, según publicaciones de Guglielmo Ronco en Turín, Italia $^{(10,11)}$, Kjaer S en Copenaghe, Dinamarca ${ }^{(12)}$, Petry en Hannover y Tubingen, Alemania ${ }^{(13)}$, Clavel C en Reims, Francia $^{(14)}$, De Sanjosé S en Barcelona, España ${ }^{(15)}$, quie- nes comunicaron resultados concordantes con la utilización del test de HPV (HC2).

En el momento del inicio de esta investigación, en diciembre de 2014, era la prueba de tamizaje disponible y comercializada en Uruguay, aprobada por la Food and Drug Administration (FDA) y la European Conformity $(\mathrm{CE})^{(16)}$.

Fue la prueba suministrada por el proyecto Estudio multicéntrico de tamizaje y triage de cáncer de cuello uterino con pruebas del virus del papiloma humano (ESTAMPA), Agencia Internacional de Investigación en Cáncer (IARC) OMS, para su uso en los países participantes del proyecto ${ }^{(17)}$.

Este test de HPV permite la detección de los HPV de alto riesgo por medio de la utilización de sondas que hibridan con los genomas de los 13 tipos de HPV de alto riesgo: $16,18,31,33,35,39,45,51,52,56,58,59$ y 68. Esta hibridación indica la presencia del HPV en la muestra y es detectada por anticuerpos específicos unidos a fosfatasa alcalina, que se ponen en evidencia mediante la emisión de luz que se registra con un luminómetro ${ }^{(9)}$.

El test de HPV no se recomienda utilizarlo en mujeres menores de 30 años por la alta frecuencia de infecciones transitorias, que lo hacen muy poco eficiente como método de tamizaje ${ }^{(9)}$.

La captura híbrida $\mathrm{HC} 2$ fue el test utilizado en varias experiencias previas que demostraron una sensibilidad muy superior a la del PAP convencional y con un valor predictivo negativo muy confiable por un lapso variable entre cinco y diez años, para mujeres de 30 años y mayores $^{(10,11,18-25)}$.

La Organización Panamericana de la Salud (OPS) ${ }^{(26)}$ sugiere que antes de la implementación del test de HPV en los programas de los diferentes países, se realice una experiencia piloto y luego de acuerdo a los resultados comenzar por una pequeña área para después ir expandiendo la cobertura de acuerdo a los resultados y la disponibilidad de recursos.

El propósito de este trabajo es analizar los primeros resultados de una experiencia piloto con la aplicación del test de HPV en una zona del departamento de Canelones, en el marco de un proyecto de investigación en colaboración con la Agencia Internacional de Investigación en Cáncer (IARC), OMS CHLCC, proyecto ESTAMPA $^{(17)}$.

\section{Material y método}

Se realizó un estudio transversal de tamizaje en el que se realizaron pruebas para la detección de HPV y Papanicolaou convencional dirigido a mujeres entre 30 y 64 años de edad asintomáticas, no embarazadas, no histerectomizadas y que no tienen antecedentes de enfermedades neoplásicas del aparato genital. 


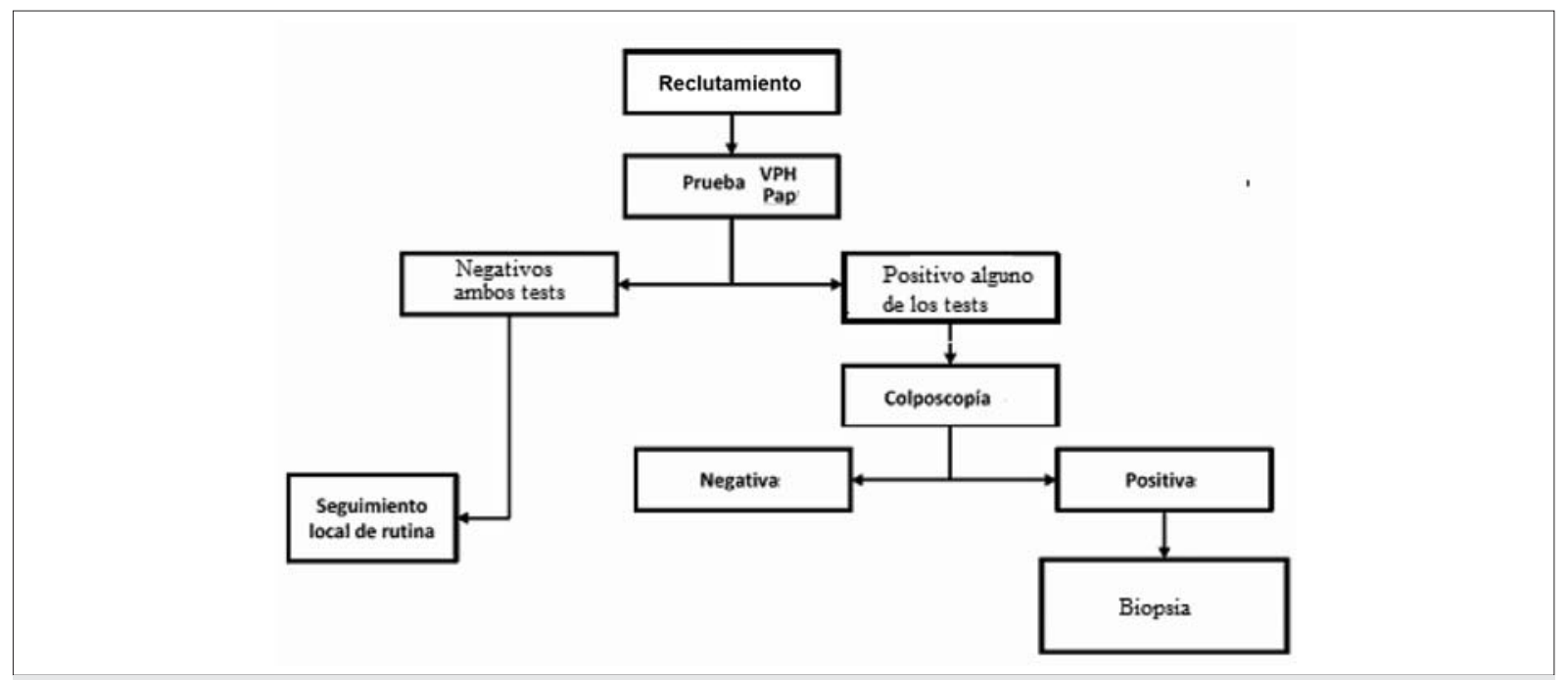

Figura 1. Flujograma.

La fecha de inicio del reclutamiento fue el 2 de diciembre de 2014 y finalizó el 30 de agosto de 2017.

\section{Área geográfica}

La investigación se llevó a cabo en el departamento de Canelones, en el área de influencia del Centro de Salud de la Costa y del Centro de Salud de Pando de la Red de Atención Primaria de Salud (RAP), ambos dependientes de la Administración de los Servicios de Salud del Estado (ASSE).

\section{Procedimiento de recolección de muestras}

En la figura 1 se detalla el flujograma seguido por las muestras.

Las usuarias fueron convocadas durante la realización de su citología de rutina. Se las invitó a participar. Se les informó del alcance y el objetivo de la investigación, y se les entregó el consentimiento informado. Si aceptó participar, se solicitó la firma del consentimiento y se las incorporó para la realización de una prueba con el fin de participar del estudio.

Desde un único procedimiento de toma de células cervicales de la usuaria con las características (que se detallan más adelante) se obtuvieron dos muestras (una para citología convencional y otra para HPV).

Con un cepillo Papette Wallach Surgical Device ${ }^{\circledR}$ se extrajo la muestra para citología convencional, se realizó extendido sobre lámina de vidrio y se fijó con una mezcla de alcohol y laca en aerosol.

El mismo cepillo se introdujo en un vial de transporte PreservCyt (ThinPrep Pap Test; Cytyc Corporation, Boxborough, Mass.) rotulado con la identificación de la usuaria, se agitó en el líquido y se descartó.
La muestra citológica se derivó para diagnóstico a los laboratorios de citología de la RAP de acuerdo a su derivación habitual, sin modificaciones para este estudio.

El vial se envió para diagnóstico HPV a temperatura ambiente, el mismo día de su extracción, al laboratorio de Biología molecular dependiente del Departamento de Patología Clínica del Centro Hospitalario Pereira Rossell (CHPR), donde se realizó el test de HPV por captura híbrida.

Ambas muestras fueron rotuladas con el mismo código que permitió identificar a la usuaria.

\section{Citología convencional}

La muestra de PAP se derivó para diagnóstico al laboratorio de citología ginecológica de la Red Integrada de Efectores Públicos de Salud (RIEPS)-RAP-ASSE, siguiendo los procedimientos habituales del PPCCU, para los cuales utilizaron la coloración de Papanicolaou para la evaluación diagnóstica.

\section{Detección del papiloma virus humano}

El test de HPV por captura híbrida HC2 DIGENE, (QIAGEN) consiste en la hibridación in vitro y amplificación de la señal mediante quimioluminiscencia de ácidos nucleicos de 13 genotipos de HPV de alto riesgo (HPV 16, 18, 31, 33, 35, 39, 45, 51, 52, 56, 58, 59, 68). Los ácidos nucleicos de HPV presentes en la muestra hibridan con una muestra de sondas específicas para HPV. Los híbridos inmovilizados reaccionan con anticuerpos específicos antihíbridos conjugados con fosfatasa alcalina. Múltiples anticuerpos se unen a los híbridos y provocan una amplificación de la señal. La emisión de luz se registró en un luminómetro y fue comparada con una señal de corte. Dado 
que todas las muestras presentan una emisión de luz mínima basal, el ensayo utiliza un calibrador que contiene 1 pg/ml de ADN de HPV-16 clonado, para determinar el punto de corte que separará los resultados negativos de los positivos. La medida de la luz emitida por la muestra se realiza en unidades relativas de luz (RLU) y se divide por la lectura del calibrador de punto de corte $(\mathrm{CO})$ para generar un resultado cuantitativo. Cuando la emisión de luz es igual o mayor al punto de corte (RLU/CO mayor o igual a 1) la prueba es positiva y eso significa que existe la presencia de alguno de los 13 tipos de HPV de alto riesgo descritos. Cuando la emisión de luz es por debajo del punto de corte (RLU/CO menor de 1) el test es negativo ${ }^{(9)}$. El test no permite identificar cuál de los tipos de HPV está presente, ni tampoco saber si se trata de una infección única o múltiple $^{(17)}$. De acuerdo al manual del fabricante, las muestras informadas con valores entre 1 y 2,5 deben repetirse $^{(9)}$.

Los resultados fueron informados al centro de salud e ingresados en una base de datos centralizada en IARC. El acceso a esta base de datos fue a través de la WEB y algunos participantes de la investigación, según su posición, tuvieron acceso mediante una clave personal que los identificó y les adjudicó determinados permisos y responsabilidades, según su nivel de gestión. Tiene respaldo permanente, seguridad y privacidad asegurados por el IARC.

\section{Derivación a colposcopía}

Las usuarias cuyos resultados del test de HPV fueron positivos se derivaron a colposcopía, al igual que las usuarias cuyo resultado de PAP fue anormal (ASC-US+). Los diagnósticos colposcópicos se realizaron en las unidades de colposcopía correspondientes dentro del sistema público de salud, en los centros que participaron de este trabajo. Los médicos colposcopistas actuantes recibieron entrenamiento previo en cuanto a la nomenclatura colposcópica, indicación de biopsias, legrados y cepillados endocervicales, así como las técnicas de tratamiento ambulatorio por parte del equipo de investigadores de IARC. De acuerdo al resultado de la colposcopía se realizaron biopsias de cuello o legrado endocervical, o ambos, para estudio histológico y confirmación diagnóstica. La indicación de biopsia o legrado endocervical se determinó a partir de la presencia de imágenes colposcópicas o resultados de PAP sospechosas de lesiones precancerosas o cáncer. A las usuarias con test HPV positivos que no presentaron imágenes colposcópicas sospechosas y el PAP fue negativo, no se les realizó biopsia.

\section{Estudio histológico}

Las biopsias y legrados endocervicales se procesaron en el laboratorio de anatomía patológica del CHPR. Se

\begin{tabular}{|c|c|c|}
\hline & Mujeres & $\%$ \\
\hline \multicolumn{3}{|l|}{ Edad } \\
\hline $30-34$ & 169 & 16,7 \\
\hline $35-39$ & 180 & 17,8 \\
\hline $40-44$ & 205 & 20,3 \\
\hline $45-49$ & 163 & 16,1 \\
\hline $50-54$ & 134 & 13,3 \\
\hline $55+$ & 159 & 15,7 \\
\hline \multicolumn{3}{|l|}{ Escolaridad } \\
\hline Primaria incompleta & 93 & 9,2 \\
\hline Primaria completa & 235 & 23,3 \\
\hline Secundaria incompleta & 489 & 48,4 \\
\hline Secundaria completa & 82 & 8,1 \\
\hline Educación superior incompleta & 44 & 4,4 \\
\hline Universidad completa & 39 & 3,9 \\
\hline Carrera técnica completa & 26 & 2,6 \\
\hline (NS)/(NR) & 2 & 0,2 \\
\hline \multicolumn{3}{|l|}{ Ocupación del jefe de hogar } \\
\hline Empleado & 343 & 34,0 \\
\hline Empleado en oficios domésticos & 177 & 17,5 \\
\hline Trabaja en un programa de empleo & 8 & 0,8 \\
\hline $\begin{array}{l}\text { Empleador (con cinco empleados o } \\
\text { menos) }\end{array}$ & 1 & 0,1 \\
\hline Profesional o técnico independiente & 50 & 5,0 \\
\hline $\begin{array}{l}\text { Trabajador independiente pero no } \\
\text { profesional }\end{array}$ & 261 & 25,8 \\
\hline Trabajo temporal & 49 & 4,9 \\
\hline Trabajo de campo & 3 & 0,3 \\
\hline Trabajador sin salario (empresa familiar) & 3 & 0,3 \\
\hline Jubilado/a & 52 & 5,2 \\
\hline Otro & 37 & 3,7 \\
\hline No responde & 26 & 2,6 \\
\hline \multicolumn{3}{|l|}{ Papanicolau previo } \\
\hline Sí & 975 & 96,5 \\
\hline No & 35 & 3,5 \\
\hline Realización del Papanicolaou & $(n=975)$ & $\%$ \\
\hline Hace menos de 2 años & 406 & 41,6 \\
\hline Entre 2 a 5 años atrás & 442 & 45,3 \\
\hline Hace más de 5 años & 127 & 13,0 \\
\hline
\end{tabular}


Tabla 2. Resultado del test de HPV y PAP en relación con la edad de la usuaria.

\begin{tabular}{lccccc}
\hline Edad & Test HPV ${ }^{*}$ & $\%$ (IC95\%) & $\begin{array}{c}\text { Citología anormal } \\
\text { (ASC-US+) }\end{array}$ & \% (IC95\%) & Total \\
\hline $30-39$ & 54 & $15,5(11,6-19,2)$ & 67 & $19,2(15,0-23,3)$ & 349 \\
$40-49$ & 45 & $12,2(8,8-15,5)$ & 72 & $19,6(15,5-23,6)$ & 368 \\
$50+$ & 27 & $9,2(5,9-12,5)$ & 28 & $9,6(6,1-12,9)$ & 293 \\
Total & 126 & $12,5(10,5-14,7)$ & 167 & $16,5(14,3-19,0)$ & 1.010 \\
\hline$* p=0,01$ & & & & & \\
$* * p=0,001$ & & & & & \\
IC: intervalo de confianza & & & & &
\end{tabular}

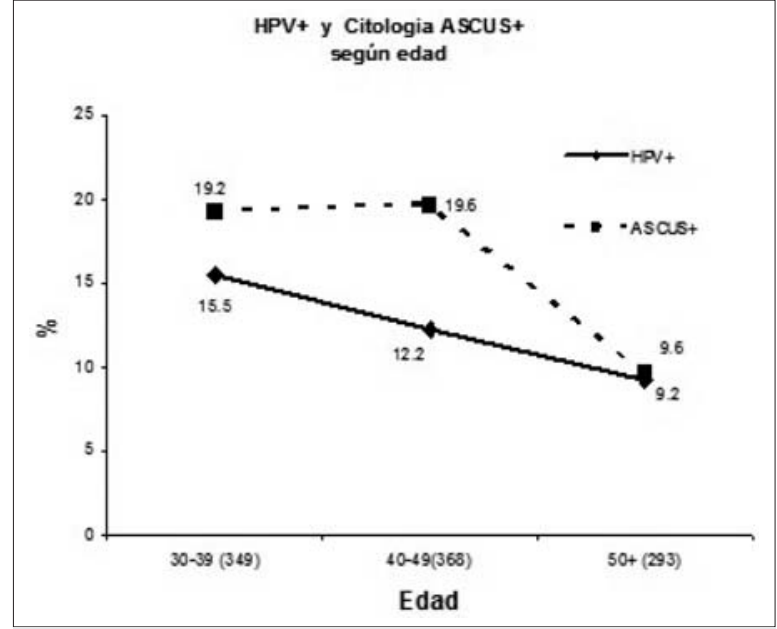

Figura 2. Resultados del test de HPV y el PAP en relación con la edad de la mujer.

realizó inmunohistoquímica P16 en los casos de H-SIL displasia moderada, según la terminología Lower Anogenital Squamous Terminology Standarization (LAST) ${ }^{(27)}$. Los técnicos anátomo patólogos recibieron entrenamiento previo al inicio del estudio en cuanto a la técnica e interpretación del P16 por parte del equipo de investigadores de IARC. Se obtuvieron muestras de todas aquellas usuarias cuyos resultados de exámenes colposcópicos indicaron la realización de la biopsia según las imágenes sospechosas de lesiones precancerosas o cáncer determinados por el colposcopista actuante.

\section{Análisis estadístico}

Se relevaron las variables: edad, educación, ocupación del jefe de hogar, la existencia de PAP previo con el intervalo desde su realización.

Para la evaluación del PAP se utilizaron las conclusiones diagnósticas según la clasificación Bethesda de-
Tabla 3. Resultados de informes de Papanicolaou.

\begin{tabular}{lcc}
\hline Resultado citología & Casos & $\%($ IC95\%) \\
\hline Negativo & 843 & $83,5(81,1-85,7)$ \\
ASC-US & 115 & $11,4(9,4-13,3)$ \\
L-SIL & 43 & $4,3(3,0-5,5)$ \\
ASC-H & 5 & $0,5(0,06-0,94)$ \\
H-SIL & 4 & $0,4(0,01-0,79)$ \\
\hline $\begin{array}{l}\text { IC: intervalo de confianza; ASC-US: células atípicas escamosas de } \\
\text { significado incierto; L-SIL: lesión intraepitelial escamosa de bajo } \\
\text { grado; ASC-H: células atípicas escamosas que no permiten descar- } \\
\text { tar H-SIL; H-SIL: lesión intraepitelial escamosa de alto grado. }\end{array}$
\end{tabular}

finidas en el formulario PPCCU publicado en el manual de procedimientos del $\mathrm{PPCCU}^{(3)}$.

Para la evaluación del test de HPV los resultados de la captura híbrida (HC2) se analizaron de acuerdo a su valor de corte, como positivo o negativo ${ }^{(9)}$.

Para los coeficientes de validez diagnóstica se usó como patrón estándar la biopsia. Aquellos casos en los cuales la biopsia no fue realizada por el colposcopista, se consideraron como negativos.

Las variables continuas se describieron con medias y desvíos estándar y las cualitativas con proporciones. Se calcularon intervalos de confianza (IC) al 95\% para las proporciones usando las aproximaciones a la distribución binomial, así como el test exacto de Fisher para las asociaciones entre variables cualitativas ${ }^{(28)}$. Para detectar la tendencia en proporciones de positividad de HPV, según la edad y la citología convencional, se usó el test de Cochran Armitage. El software usado fue Stata v15.

\section{Consideraciones éticas}

La presente investigación ha sido aprobada por los comités de ética de IARC, OPS, CHPR, Instituto Nacional 
Tabla 4. Resultado del test de HPV según informe citológico.

\begin{tabular}{|c|c|c|c|c|c|}
\hline Citología & $H P V-$ & $\%$ (IC95\%) & HPV+ & $\%$ (IC95\%) & Total \\
\hline Negativo & 757 & $89,8(87,6-91,8)$ & 86 & $10,2(8,2-12,4)$ & 843 \\
\hline ASC-US & 99 & $86,1(78,4-91,8)$ & 16 & $13,9(8,2-21,6)$ & 115 \\
\hline L-SIL & 24 & $55,8(39,9-70,9)$ & 19 & $44,2(29,1-60,1)$ & 43 \\
\hline ASCH-H-SIL & 4 & $44,4(13,7-78,8)$ & 5 & $55,6(21,2-86,3)$ & 9 \\
\hline Total & 884 & $87,5(85,3-89,5)$ & 126 & $12,5(10,5-14,7)$ & 1.010 \\
\hline
\end{tabular}

Tabla 5. Resultado del test de HPV en usuarias con informe citológico de ASC-US según edad.

\begin{tabular}{|lccccc}
\hline Edad & HPV- & $\%(I C 95 \%)$ & HPV+(\%) & $\%$ (IC95\%) & Total \\
\hline $30-39$ & 38 & $80,9(66,8-90,9)$ & 9 & $19,1(9,1-33,2)$ & 47 \\
$40-49$ & 40 & $87,0(73,8-95,1)$ & 6 & $13,0(4,9-26,2)$ & 46 \\
$50+$ & 21 & $95,5(77,2-99,9)$ & 1 & $4,5(0,1-22,8)$ & 22 \\
Total & 99 & $86,1(78,4-91,8)$ & 16 & $13,9(8,2-21,6)$ & 115 \\
\hline & & & & \\
ASC-US: células atípicas escamosas de significado incierto.
\end{tabular}

Tabla 6. Resultado histológico de la biopsia en relación con el test de HPV.

\begin{tabular}{lccc}
\hline Biopsia & \multicolumn{2}{c}{ Test HPV } & Total \\
\cline { 2 - 3 } & Negativo & Positivo & \\
\hline Negativo* & 884 & 113 & 997 \\
CIN2+ & 0 & 13 & 13 \\
Total & 884 & 126 & 1.010 \\
\hline
\end{tabular}

Sensibilidad \% (IC 95\%) $\quad 100,0(75,2-100,0)$

Especificidad \% (IC 95\%) 88,7 (86,5-90,5)

VPP \% (IC 95\%) 10,3 $\quad$ (5,6-16,9)

VPN \% (IC 95\%) $\quad 100,0(99,5-100,0)$

*Incluye resultados negativos y aquellos que no ameritaban biopsia según el examen de colposcopía.

de Trasplantes de Órganos y Tejidos y MS, a los cuales se les brinda información periódica sobre modificaciones al proyecto original aprobado.

\section{Resultados}

Se captaron 1.010 mujeres provenientes del área de influencia de las policlínicas del prestador ASSE de Ciudad de la Costa y Pando, en el departamento de Canelones, que forman parte del área metropolitana de la capital de Uruguay, Montevideo.

En la tabla 1 se puede apreciar la distribución por edades (media 44,2 desvío estándar: 8,9). La totalidad de las mujeres manifestaron saber leer y escribir, predominando en el grupo las usuarias con nivel de escolaridad de secundaria incompleta. El 16,3\% de ellas declararon tener por lo menos 12 años de escolaridad (secundaria completa y universidad).

En cuanto a la situación actual de los jefes de hogar, $846(83,8 \%)$ trabajan en el momento de la entrevista, mientras que $49(4,9 \%)$ están desempleados en búsqueda de trabajo y $52(5,2 \%)$ están jubilados. Las ocupaciones predominantes de los jefes de hogar son empleados $343(34 \%)$, trabajador independiente no profesional ( $\sin$ contrato formal de trabajo) $261(25,8 \%)$ y empleado en oficios domésticos 177 (17,5\%). En referencia al acceso al agua potable por tubería, $978(96,8 \%)$ manifestaron tenerlo y $995(98,5 \%)$ tienen baño con sanitario con arrastre de agua. 
Tabla 7a. Resultado de la biopsia en relación con la citología convencional considerando punto de corte en ASC-US.

\begin{tabular}{llll}
\hline \multirow{3}{*}{ Biopsia } & Total \\
\cline { 2 - 2 } & Negativo & AS-CUS+ \\
\hline
\end{tabular}

\begin{tabular}{|c|c|c|c|}
\hline Negativo* & 837 & 160 & 997 \\
\hline $\mathrm{CIN} 2+$ & 6 & 7 & 13 \\
\hline Total & 843 & 167 & 1010 \\
\hline Sensibilidad \% (IC 95\%) & \multicolumn{2}{|c|}{$54,0(25,1-80,7)$} & \\
\hline Especificidad \% (IC 95\%) & \multicolumn{2}{|c|}{$84,0(56,5-81,5)$} & \\
\hline VPP \% (IC 95\%) & \multicolumn{2}{|c|}{$4,0(1,7-8,4)$} & \\
\hline VPN \% (IC 95\%) & \multicolumn{2}{|c|}{$99,0(98,4-99,7)$} & \\
\hline
\end{tabular}

*Incluye resultados negativos y aquellos que no ameritaban biopsia según el examen de colposcopía; ASC-US: células atípicas escamosas de significado incierto; ASCUS+: incluye diagnósticos ASC-US, L-SIL, ASC-H, H-SIL, carcinoma in situ y cáncer.

Las comodidades que tienen en el domicilio: 871 $(86,2 \%)$ usuarias manifestaron tener lavarropas; 608 $(60,2 \%)$ horno microondas; $358(55,2 \%)$ televisión con cable; 365 (36,1\%) computadora portátil, y $242(24 \%)$ automóvil.

Con respecto a la realización de Papanicolaou alguna vez en la vida, $975(96,5 \%)$ contestaron en forma afirmativa.

\section{Resultado del test de HPV}

Con respecto al resultado del test de HPV la totalidad de las muestras extraídas fueron aptas para el diagnóstico. El resultado fue positivo en $126(12,5 \%$, IC95\% $10,5-14,7)$ de las usuarias con mayor porcentaje de resultados positivos en el grupo de 30 a $39(15,5 \%$, IC95\% 11,6-19,2) años descendiendo a 9,2\% (IC95\% $5,9-12,5)$ a los 50-64 años $(\mathrm{p}=0,01)$ (tabla 2, figura 2).

\section{Resultado de la citología convencional (test de Papanicolaou)}

El PAP fue anormal en 167 (16,5\%, IC95\% 14,3-19,0) casos con diagnóstico ASC-US y mayores (ASC-US+), siendo más frecuentes entre mujeres de 40 a 49 años. La proporción de PAP alterados disminuye según la edad de 19,2\% (IC95\% 15,0-23,3) en aquellas mujeres de 30 a 39 años a 9,6\% (IC95\% 6,1-12,9) en las mujeres de 50 y más años $(\mathrm{p}=0,001)$ (tabla 2 , figura 2$)$.

En la distribución de los resultados de los informes de citología se puede observar que el resultado del Papanicolaou fue negativo para malignidad o lesión intraepi-
Tabla 7b. Resultado de la biopsia en relación con la citología convencional considerando punto de corte en L-SIL.

\begin{tabular}{|lccc}
\hline \multirow{2}{*}{ Biopsia } & \multicolumn{2}{c}{ Citología } & Total \\
\cline { 2 - 3 } & Negativo & L-SIL+ & \\
\hline Negativo* & 951 & 46 & 997 \\
CIN2+ & 7 & 6 & 13 \\
Total & 958 & 52 & 1010 \\
\hline Sensibilidad \% (IC 95\%) & $46,0(19,2-74,8)$ & \\
Especificidad \% (IC 95\%) & $95,0(93,8-96,6)$ & \\
VPP \% (IC 95\%) & 12,0 (4,3-23,4) \\
VPN \% (IC 95\%) & 99,0 (98,5-99,7) \\
\hline & \\
* Incluye resultados negativos y aquellos que no ameritaban biop- \\
sia según el examen de colposcopía; L-SIL + incluye diagnósticos \\
L-SIL, ASC-H, H-SIL, carcinoma in situ y cáncer.
\end{tabular}

telial en $843(83,5 \%$, IC95\% 81,1-85,7). Mientras que de los 167 casos anormales $(16,5 \%)$, la presencia de diagnóstico ASC-US se diagnosticó en 115 casos $(11,4 \%$, IC95\% 9,4-13,3) (tabla 3).

De acuerdo al resultado de Papanicolaou se encontró una mayor proporción de resultados positivos de HPV a medida que aumenta la gravedad del resultado citológico desde ASC-US a L-SIL (L-SIL: lesión intraepitelial escamosa de bajo grado) hasta ASC-H/H-SIL (ASC-H: células atípicas escamosas que no pueden descartar H-SIL), desde 10,2\% (IC95\% 8,1-12,2) hasta 55,6\% (IC95\% 23,1-88,0), ( $<<0,001)$ (tabla 4).

Considerando a las usuarias con diagnóstico de ASC-US, se observó una disminución de la frecuencia de HPV+ a medida que avanza la edad, pero no estadísticamente significativa $(\mathrm{p}=0,10)$ (tabla 5).

\section{Diagnóstico histológico}

De acuerdo al resultado obtenido mediante biopsia dirigida por colposcopía o legrado endocervical se diagnosticaron 13 lesiones precursoras del cáncer de cuello uterino (4 CIN2 y 9 CIN3), (CIN3 = neoplasia intraepitelial cervical grado 3$)$.

Todas ellas tuvieron un resultado del test de HPV positivo (100\%, IC95\% 75,2-100,0). O sea que encontramos una sensibilidad para CIN2+ de 100\% (IC95\% $75,2-100,0)$ con el test de HPV, con un valor predictivo negativo de $100 \%$ (IC95\% 99,5-100,0) y un valor predictivo positivo de $10,3 \%$ (IC95\% 5,6-16,9). Se requi- 
rieron 126 casos positivos de HPV para detectar los 13 casos de CIN 2+ (tabla 6).

La sensibilidad para Papanicolaou fue de 54\% (IC95\% 25,1-80,7) si tomamos en cuenta el PAP anormal como ASC-US+ y desciende a 46,0\% (IC95\% 19,2-74,8) si consideramos el Papanicolaou anormal como L-SIL+ (tabla 7a y 7b).

Presentaron un resultado de Papanicolaou patológico uno de cuatro CIN2 $(25,0 \%$, IC $95 \% 0,63-80,5)$ y en cinco de nueve CIN3 $(55,6 \%$, IC95\% 21,0-86,0), siendo siete en trece CIN2+ $(54,0 \%$, IC95\% 25,0-80,0).

Por otro lado, el valor predictivo positivo resultó de 4,0\% (IC 95\% 1,7-8,4) considerando al PAP anormal como ASC-US+ y de 12,0\% (IC95\% 4,3-23,4) considerando al PAP anormal como L-SIL+.

\section{Discusión}

Se trata de una primera experiencia con la incorporación del test de HPV como tamizaje primario del cáncer de cuello uterino, en comparación con el test de Papanicolaou convencional, que se realizó en una población perteneciente al PPCCU de Uruguay.

Algunos de los factores que permitieron la uniformidad diagnóstica de la muestra analizada fue que todos los informes de Papanicolaou se realizaron en el laboratorio de citología ginecológica de la Red Integrada de Efectores Públicos en Salud (RIEPS-RAP-ASSE), así como la totalidad de los informes histológicos de las biopsias, legrados endocervicales y piezas de conización se procesaron y diagnosticaron en el laboratorio de anatomía patológica en el CHPR.

Por otro lado, un factor limitante para la representatividad es que la población pertenecía a un área del departamento de Canelones, y, por lo tanto, no fue representativa de la totalidad de la población de Uruguay.

El tamaño de la muestra de 1.010 mujeres se consideró aceptable como para una experiencia piloto, a pesar de la baja prevalencia de las lesiones precancerosas en la población de Uruguay.

La positividad para la prueba de HPV que correspondió a los tipos virales de alto riesgo fue de $12,5 \%$ de los casos, siendo similar a los resultados encontrados con el mismo método y los mismos grupos etarios dentro del trabajo multicéntrico de investigación de otras regiones de Latinoamérica, según datos preliminares del proyecto ESTAMPA IARC-OMS. Utilizando el mismo test de HPV, Arrossi y colaboradores encontraron en Jujuy (Argentina) un porcentaje de positividad de $12 \%$ en mujeres mayores de 30 años, tanto cuando se realizó mediante autotoma o con la muestra extraída por un clínico $^{(29)}$.

Estos resultados fueron numéricamente inferiores a los obtenidos en un estudio previo de Berois y colabora- dores $^{(30)}$. El objetivo de esa investigación previa fue identificar los tipos de HPV circulantes, para evaluar el potencial beneficio de la aplicación de la vacuna contra el HPV en Uruguay. En esa oportunidad el test utilizado fue PCR (reacción de polimerización en cadena) Papillocheck $^{\mathrm{R}(31)}$ (Greiner bio one, Alemania). Este test de PCR Papillocheck ${ }^{R}$ identifica a 18 tipos de HPV de alto riesgo. El test de captura híbrida, utilizado en esta investigación, detecta la presencia de 13 tipos de HPV de alto riesgo, sin identificarlos. La muestra poblacional de la publicación previa de Berois y colaboradores $^{(30)}$ fue representativa del país, proveniente de diez departamentos de Uruguay especialmente seleccionados. En ese trabajo ${ }^{(30)}$ se encontró $18 \%$ de positividad de los tipos de alto riesgo de HPV en mujeres asintomáticas no embarazadas, no histerectomizadas, de 30 años y mayores que asistieron espontáneamente a los centros de salud públicos de ASSE. Esta diferencia en la positividad de $12,5 \%$ en el trabajo actual, entre otros factores, probablemente se explique por tener ambos estudios muestras de población diferentes y técnicas diferentes. La sensibilidad clínica de la HC2 para CIN2+ es similar al PCR, aunque los valores informados de carga viral no sean concordantes, como ha sido descrito en forma previa $^{(32)}$. La mayor carga viral aumenta la sensibilidad del test para CIN2+, solo para HPV 16, considerada en forma individual, como lo demostraron otros investigadores $^{(33-35)}$.

En el presente trabajo se encontró un mayor porcentaje de mujeres HPV+ en el grupo de 30 a 39 años con un gradual descenso según aumentaba la edad, no encontrándose un nuevo incremento en la positividad en el grupo de mujeres mayores de 50 años como el informado en otros estudios ${ }^{(36,37)}$.

Con respecto a los resultados de la citología convencional se encontró $16,5 \%$ de resultados anormales (ASC-US+). El diagnóstico de células atípicas escamosas de significado incierto (ASC-US) es una amplia categoría en citología ginecológica que comprende diferentes procesos etiológicos que incluyen la infección $\mathrm{HPV}$, neoplasias malignas y cambios reactivos ${ }^{(38)}$, representando el 11,4\% de todos los informes de Papanicolaou de las usuarias estudiadas y contribuyendo con el $68,9 \%$ de los informes patológicos de PAP.

Siendo este un criterio habitual de derivación a colposcopía en la práctica en el país, aunque no recomendada por las Guías de práctica clínica del tamizaje del cáncer de cuello de útero ${ }^{(39)}$, esto puede explicar que el número de colposcopías derivadas por indicación del PAP $(16,5 \%)$ fue mayor que por el resultado de un HPV positivo (12,5\%). Pese a esta diferencia, los diagnósticos ASC-US no detectaron mayor número de casos CIN2+ que el test de HPV. Si se dispusiera del test de HPV de 
acuerdo a las guías nacionales de tamizaje del cáncer de cuello uterino en Uruguay ${ }^{(39)}$, se podría evitar la derivación a colposcopía de un número importante de estas mujeres con resultado citológico de ASC-US.

Según este estudio el porcentaje de positividad para el test de HPV en los casos de ASC-US fue de 13,9\%, siendo mayor en el grupo más joven $(19,1 \%)$, disminuyendo en edades más avanzadas. Esta relación con la edad de la paciente también fue comunicada por otros investigadores $^{(40)}$, pero se han publicado porcentajes mayores de mujeres HPV positivas en aquellas muestras con resultado de ASC-US ${ }^{(41-45)}$.

Este hallazgo nos estaría indicando un alto número de resultados con sobrediagnóstico de PAP, que generan más demanda de colposcopías y biopsias que no se reflejan en una mayor detección de lesiones premalignas.

La positividad del test de HPV hallado en la presente investigación está en relación directa con el mayor grado de lesión intraepitelial en la citología convencional. El porcentaje de positividad va en aumento desde el 10,2\% en los extendidos negativos a 55,6\% en el H-SIL, similar a lo encontrado por estudios previos en Uruguay ${ }^{(30)}$.

Una de las dificultades que se han planteado en otros países en la incorporación del test de HPV en los programas de prevención del cáncer de cuello uterino ha sido el temor al aumento del número de exámenes colposcópicos a los cuales no se les pudiera dar respuesta ${ }^{(46)}$. Los resultados hallados en este trabajo muestran una situación diferente, con un número mayor de colposcopías como consecuencia del diagnóstico de citologías anormales, sobre todo a expensas del informe de ASC-US en el PAP que las que serían indicadas por un resultado del test de HPV+.

Si analizamos la relación entre el resultado histológico obtenido mediante la biopsia dirigida por colposcopía y el resultado del test de Papanicolaou, nos encontramos que el PAP fue patológico en siete de trece de diagnósticos histológicos de lesiones precursoras del cáncer de cuello uterino, resultando en una sensibilidad para la citología de 54,0\% (IC 95\% 25,1-80,7), con una especificidad de 84,0\% (56,5-81,5). Si cambiamos el valor de corte para el PAP anormal a L-SIL, mejora la especificidad a 95,0\% (93,8-96,6), pero desciende el valor de la sensibilidad a 46,0\% (IC 95\% 19,2-74,8). La baja sensibilidad hallada para la citología para la captación de lesiones CIN2+ está dentro de los valores publicados, $51 \%{ }^{(10)}$ y $53 \%{ }^{(11)}$.

Con respecto a la relación entre el resultado histológico obtenido mediante la biopsia dirigida por colposcopía y el resultado del test de HPV, el test de HPV fue positivo en 13 de 13 usuarias diagnosticadas con CIN2+, correspondiendo a una sensibilidad de $100,0 \%(75,2-100,0)$ y una espe- cificidad de 88,7\% $(86,5-90,5)$, mayor que para el PAP. Con respecto a este último indicador los diferentes estudios muestran resultados diversos, en algunos la especificidad del PAP ha sido mayor que el test de $\mathrm{HPV}^{(47)}$, así como en otros ha sido similar ${ }^{(48)(49)}$. Los valores de especificidad se deben tomar con precaución, dado que no se realizó biopsia a los resultados negativos a ambas pruebas, ni aquellas cuya colposcopía resultaba negativa, debido a objeciones de carácter ético planteadas en forma previa, lo que puede introducir cierto sesgo de verificación.

Nuestros resultados muestran una mayor eficacia en la detección de lesiones precursoras del cáncer de cuello uterino con el test de HPV como test de tamizaje primario que el PAP convencional, cuando se aplica dentro de un programa de salud público, en acuerdo a lo comunicado por otros investigadores. Ronco y colaboradores ${ }^{(10,11,50)}$ reportaron que el test de HPV-HC2 tuvo una sensibilidad 50\% mayor que el PAP convencional para CIN2+ y una protección 60\%-70\% mayor para el cáncer invasor que el PAP convencional ${ }^{(51,52)}$.

\section{Conclusión}

Los resultados obtenidos en este trabajo nos muestran que la aplicación del test de HPV captura híbrida fue más eficaz que el PAP como tamizaje primario del cáncer de cuello uterino en cuanto a que fue positivo en el $100 \%$ de los diagnósticos histológicos de lesiones precursoras del cáncer de cuello uterino diagnosticadas.

Pese al pequeño tamaño muestral, el cual no permite encontrar un número alto de casos patológicos confirmados por biopsia, se pudo apreciar que el test de HPV fue más eficiente que el Papanicolaou desde el punto de vista de la derivación de usuarias a colposcopía, en las condiciones habituales de funcionamiento en las policlínicas de ASSE estudiadas en esta investigación, que son parte del programa de prevención del cáncer de cuello uterino en Uruguay.

La incorporación del test de HPV como test de tamizaje primario para la prevención del cáncer de cuello uterino, de acuerdo a las recomendaciones de OPS en el Manual para Gerentes de Programas de Salud, sería una medida deseable para lograr una mayor captación de lesiones precursoras del cáncer de cuello uterino, como lo sugieren los resultados de este trabajo para nuestra población.

También debería insistirse en el potencial beneficio de la implementación de controles de calidad de la lectura de láminas de Papanicolaou en los laboratorios del PPCCU en Uruguay.

Estos resultados estarían justificando la realización de un estudio costo efectividad y un ensayo de implementación poblacional en un área mayor del programa 
de prevención del cáncer de cuello uterino, para la eventual aplicación del test de HPV como test de tamizaje primario para la prevención del cáncer de cuello uterino en Uruguay.

\section{Agradecimientos}

Agradecemos al Programa de Reproducción Humana de la Organización Mundial de la Salud por su apoyo financiero en la ejecución del estudio ESTAMPA

\section{Abstract}

Introduction: in Uruguay, cervical cancer occupied the third place with an average incidence of 312 new cases per year. 134 women died in the 2010-2014 period.

Objective: the study aims to analyse the first results in a pilot experience with the application of Hybrid Capture ${ }^{\circledR} 2$ High-Risk HPV DNA Test ${ }^{\mathrm{TM}}$ (hc2) (Qiagen) as the primary screening test for cervical cancer, in the Department of Canelones.

Method: 1.010 asymptomatic women whose ages ranged between 30 and 64 years old were studied when they requested a pap smear at two units of sample taking within the Cervical Cancer Prevention Program.

Samples were taken along with the pap smear, and sent to one of the cytology labs in the primary health care network, the HPV sample being processed with the hybrid capture technique in the molecular biology laboratory of the Pereira Rossell Hospital Center. Users with abnormal HPV+ and abnormal pap smear results (ASCUS+) were referred to colposcopy, with subsequent biopsy and treatment if required.

Results: HPV test was positive in 126/1010 women $(12.5 \%)$ and PAP was abnormal in $167 / 1010$ cases (16.5\%). HPV test was positive in all cases CIN2+ 13/13 $(100 \%)$ whereas PAP was abnormal (ASCUS+) in $7 / 13$ $54 \%$ ) for CIN2+ in biopsy.

Conclusion: HPV test was more effective and efficient than pap smear to detect pre-cancer lesions in the cervix.

\section{Resumo}

Introdução: no Uruguai, no período 2010 - 2014, o câncer de colo do útero foi a terceira causa com uma incidência média de 312 novos casos por ano e uma média de 134 mortes por ano.

Objetivo: o objetivo deste trabalho foi analisar os primeiros resultados de uma experiência piloto com a utilização do exame de HPV Captura Híbrida HC2 (Qiagen R ) na tamizagem primária do câncer de colo do útero em una zona do departamento de Canelones.

Método: foram estudadas 1010 mulheres assintomáticas, com idades entre 30 e 64 anos que foram a duas unidades do Programa de Prevenção do Câncer do Colo do Útero para a coleta de amostra para exame de Papanicolau (PAP).

Realizou-se uma única extração para duas amostras: uma para PAP, que foi encaminhada a um laboratório de citologia da rede de atenção primária de saúde e outra para o exame de HPV que foi processada com a técnica de captura híbrida no laboratório de biologia molecular do Centro Hospitalario Pereira Rossell. As usuárias com resultados HPV + e PAP anormais (ASCUS+) foram encaminhadas para realização de colposcopia, com biopsia e tratamento quando fosse necessário.

Resultados: o exame de HPV foi positivo em $126 / 1010$ mulheres $(12.5 \%)$ e o PAP foi anormal em 167/1010 (16.5\%). O exame de HPV foi positivo em todos los casos CIN2+ 13/13 (100\%) e o PAP foi anormal (ASCUS+) em 7/13 (54\%) para CIN2+ por biopsia.

Conclusão: o exame de HPV foi mais eficaz e eficiente que o PAP para detecção de lesões pré-cancerosas de colo do útero.

\section{Bibliografía}

1. International Agency for Research on Cancer (IARC). Global Cancer Observatory 2012. Disponible en: https://gco.iarc.fr/ [Consulta: 24 marzo 2019].

2. Comisión Honoraria de Lucha Contra el Cáncer (CHLCC). Registro Nacional de Cáncer. Situación epidemiológica del Uruguay en relación al cáncer-Mayo 2019. Disponible en: http://www.comisioncancer.org.uy/uc_513_ 1.html [Consulta: 25 marzo 2019].

3. Rodríguez G, Alonso R, Ortiz de Taranco M. Programa de prevención de cáncer de cuello uterino en el Uruguay "Dr. Enrique Pouey": estrategia y manual de procedimientos. Montevideo: CHLCC, 2007:129 p.

4. Muñoz N, Bosch FX, de Sanjosé S, Herrero R, Castellsagué X, Shah KV, et al. Epidemiologic classification of human papillomavirus types associated with cervical cancer. $\mathrm{N}$ Engl J Med 2003; 348(6):518-27.

5. Bosch FX, Lorincz A, Muñoz N, Meijer CL, Shah KV. The causal relation between human papillomavirus and cervical cancer. J Clin Pathol 2002; 55(4):244-65.

6. Walboomers JM, Jacobs MV, Manos MM, Bosch FX, Kummer JA, Shah KV, et al. Human papillomavirus is a necessary cause of invasive cervical cancer worldwide. J Pathol 1999; 189:12-9.

7. Ostör AG. Natural history of cervical intraepithelial neoplasia: a critical review. Int J Gynecol Pathol 1993; 12(2): 186-92.

8. Organización Panamericana de la Salud. Control integral del cáncer cervicouterino: guía de prácticas esenciales. $2^{\mathrm{a}}$ ed Wahington, DC: OPS, 2016. Disponible en: http://iris.paho. org/xmlui/bitstream/handle/123456789/28512/9789275318 799 spa.pdf?ua=1 [Consulta: 27 marzo 2019].

9. Qiagen NV. Digene HC2 HPV DNA Test (5196-1330 19300) Disponible en: https://www.qiagen.com/mq/ 
search.aspx?q=REF\%205196-1330\%2019300\#\&\&pg=1

[Consulta: 26 marzo 2019].

10. Ronco G, Giorgi-Rossi P, Carozzi F, Confortini M, Dalla Palma P, Del Mistro A, et al. Results at recruitment from a randomized controlled trial comparing human papillomavirus testing alone with conventional cytology as the primary cervical cancer screening test. J Natl Cancer Inst 2008; 100(7):492-501.

11. Ronco G, Giorgi-Rossi P, Carozzi F, Confortini M, Dalla Palma P, Del Mistro A, et al. Efficacy of human papillomavirus testing for the detection of invasive cervical cancers and cervical intraepithelial neoplasia: a randomised controlled trial. Lancet Oncol 2010; 11:249-57.

12. Kjaer SK, van den Brule AJ, Paull G, Svare EI, Sherman ME, Thomsen BL, et al. Type specific persistence of high risk human papillomavirus (HPV) as indicator of high grade cervical squamous intraepithelial lesions in young women: population based prospective follow up study. BMJ 2002; 325(7364):572.

13. Petry KU, Menton S, Menton M, van Loenen-Frosch F, de Carvalho Gomes H, Holz B, et al. Inclusion of HPV testing in routine cervical cancer screening for women above 29 years in Germany: results for 8466 patients. Br J Cancer 2003; 88:1570-7.

14. Clavel C, Masure M, Bory JP, Putaud I, Mangeonjean C, Lorenzato $\mathbf{M}$, et al. Human papillomavirus testing in primary screening for the detection of high-grade cervical lesions: a study of 7932 women. Br J Cancer 2001; 84:1616-23.

15. de Sanjose S, Almirall R, Lloveras B, Font R, Diaz M, Muñoz N, et al. Cervical human papillomavirus infection in the female population in Barcelona, Spain. Sex Transm Dis 2003; 30:788-93.

16. Schiffman M, Wentzensen N, Wacholder S, Kinney W, Gage JC, Castle PE. Human papillomavirus testing in the prevention of cervical cancer. J Natl Cancer Inst 2011; 103(5):368-83.

17. Almonte M. EStudio multicéntrico de TAMizaje y triaje usando la prueba de PApilomavirus humano (ESTAMPA). Disponible en: https://www.paho.org/hq/index.php?option $=$ com_docman\&view=download\&category_slug=experiencias-implementacion-programas-tamizaje-cacu-basados-prueba-vph-31jul-1ago-2018-washington-dc-9978\&alias $=46029$-estudio-multicentrico-tamizaje-triaje-usando-prueba-papilomavirus-humano-estampa-maribel-almonte-iarc-029\&Itemid $=270 \&$ lang $=$ en $\quad[$ Consulta: 3 setiembre 2017].

18. Bulkmans NW, Berkhof J, Rozendaal L, van Kemenade J, Boeke A, Bulk S, et al. Human papillomavirus DNA testing for the detection of cervical intraepithelial neoplasia grade 3 and cancer: 5-year follow-up of a randomised controlled implementation trial. Lancet 2007; 370(9601):1764-72.

19. Kitchener HC, Gilham C, Sargent A, Bailey A, Albrow R, Roberts C, et al. A comparison of HPV DNA testing and liquid based cytology over three rounds of primary cervical screening: extended follow up in the ARTISTIC trial. Eur J Cancer 2011; 47(6):864-71.
20. Kotaniemi-Talonen L Nieminen P, Anttila A, Hakama M. Routine cervical screening with primary HPV testing and cytology triage protocol in a randomised setting. $\mathrm{Br} \mathrm{J}$ Cancer 2005; 93(8):862-7.

21. Leinonen M, Nieminen $\mathbf{P}$, Kotaniemi-Talonen L, Malila $\mathbf{N}$, Tarkkanen J, Laurila P, et al. Age-specific evaluation of primary human papillomavirus screening vs conventional cytology in a randomized setting. J Natl Cancer Inst 2009; 101(23):1612-23.

22. Naucler P, Ryd W, Törnberg S, Strand A, Wadell G, Elfgren K, et al. Human papillomavirus and Papanicolaou tests to screen for cervical cancer. N Engl J Med 2007; 357(16):1589-97.

23. Naucler P, Ryd W, Törnberg S, Strand A, Wadell G, Elfgren K, et al. Efficacy of HPV DNA testing with cytology triage and/or repeat HPV DNA testing in primary cervical cancer screening. J Natl Cancer Inst 2009; 101(2):88-99.

24. Rijkaart DC, Berkhof J, Rozendaal L, van Kemenade FJ, Bulkmans NW, Heideman DA, et al. Human papillomavirus testing for the detection of high-grade cervical intraepithelial neoplasia and cancer: final results of the POBASCAM randomised controlled trial. Lancet Oncol 2012; 13(1):78-88.

25. Ronco G, Arbyn M, Meijer CJLM, Snijders PJF, Cuzick J. Screening for cervical cancer with primary testing for human papillomavirus. En: Anttila A, Arbyn A, De Vuyst H, Dillner J, Dillner L, Franceschi S, et al, eds. European guidelines for quality assurance in cervical cancer screening. Supplements. 2a ed. Luxembourg: Office of the European Union, 2015:1-59. Disponible en: https://www.gisci.it/documenti/news/EW0115451ENN_002.pdf [Consulta: 26 marzo 2019].

26. Organización Panamericana de la Salud. Incorporación de la prueba del virus del papiloma humano en programas de prevención de cáncer cervicouterino. Manual para gerentes de programas de salud. Washington, OPS, 2016. Disponible en: https://www.paho.org/hq/index.php?option=com_docman\&view=download\&category_slug=guias-manuales-3444\&alias=36609-incorporacion-prueba-virus-papiloma-humano-programas-prevencion-cancer-cervicouterino-manual-gerentes-programas-salud-609\&Itemid=270\&lang=en [Consulta: 26 marzo 2019].

27. Darragh TM, Colgan TJ, Cox JT, Heller DS, Henry MR, Luff RD, et al. The lower anogenital squamous terminology standardization project for HPV-associated lesions: background and consensus recommendations from de College of American Pathologists and the American Society for Colposcopy and Cervical Pathology. J Low Genit Tract Dis 2012; 16(3):205-42.

28. Brown LD, Cai TT, DasGupta A. Interval estimation for a binomial proportion: rejoinder. Statist Sci 2001; 16(2):128-.

29. Arrossi S, Thouyaret L, Herrero R, Campanera A, Magdaleno A, Cuberli M, et al. Effect of self-collection of HPV DNA offered by community health workers at home visits on uptake of screening for cervical cancer (the EMA study): a population-based cluster-randomised trial. Lancet Glob Health 2015; 3(2):e85-94. 
30. Berois N, Heard I, Fort Z, Alonso R, Sica A, Moerzinger P, et al. Prevalence of type-specific HPV infection in Uruguay. J Med Virol 2014; 84(4):647-52.

31. Papillocheck ${ }^{\circledR}$. Manual de Instrucciones REF 465 060. (Revisión: BQ-013-07) Frickenhausen, Deu: Greiner Bio-One, 2011:44 p. Disponible en: https://www.gbo.com/fileadmin/user_upload/Downloads/IFU_Instruc-

tions_for_Use/IFU_Diagnostics/PapilloCheck/IFU_Papi-

1loCheck_Rev._BQ-013-07_ES.pdf [Consulta: 24 marzo 2019].

32. IARC Working Group on the Evaluation of Carcinogenic Risks to Humans. Human Papillomaviruses. Lyon, : 2007. (IARC monographs on the evaluation of carcinogenic risks to humans; v.90). Disponible en: https://monographs.iarc.fr/ wp-content/uploads/2018/06/mono90.pdf [Consulta: 27 marzo 2019].

33. Gravitt P, Kovacic M, Herrero R, Schiffman M, Bratti C, Hildesheim A, et al. High load for most high human papillomavirus genotypes is associated with prevalent cervical cancer precursors but only HPV 16 load predicts the development of incident disease. Int $\mathrm{J}$ Cancer 2007; 121(12): 2787-93.

34. Lorincz A, Castle P, Sherman M, Scott D, Glass A, Wacholder S, et al. Viral load of human papillomavirus and risk of CIN3 or cervical cancer. Lancet 2002; 360(9328):228-9.

35. Kinney W, Stoler M, Castle P. Special commentary: patient safety and the next generation of HPV DNA tests. Am J Clin Pathol 2010; 134(2):193-9.

36. Ramas V, Mirazo S, Bonilla S, Mendoza L, Lago O, Basiletti J, et al. Human papillomavirus genotypes distribution in cervical samples from Uruguayan women. J Med Virol 2013; 85(5):845-51.
37. de Sanjosé S, Diaz M, Castellsagué X, Clifford G, Bruni L, Muñoz N, et al. Worldwide prevalence and genotype distribution of cervical human papillomavirus DNA in women with normal cytology: a meta-analysis. Lancet Infect Dis 2007; 7(7):453-9.

38. Solomon D, Nayar R, eds. The Bethesda system for reporting cervical cytology. Definitions, criteria, and explanatory notes. New York: Springer-Verlag, 2004.

39. Uruguay. Ministerio de Salud Pública. Guía de práctica clínica de tamizaje de cáncer de cuello de útero. Montevideo: MSP, 2005:39 p.

40. Shiffman M, Solomon D. Findings to date from the ASCUS-LSIL Triage Study (ALTS). Arch Pathol Lab Med 2003; 127(8):946-9.

41. Tworek J, Jones B, Raab S, Clary K, Wolsh M. The value of monitoring human papillomavirus DNA results of Papanicolaou tests diagnosed as atypical squamous cells of undetermined significance: a College of American Pathologists Q-Probes study of 68 institutions. Arch Pathol Lab Med 2007; 131(10):1525-31.

42. Davey D, Woodhouse S, Styer P, Stastny J, Mody D. Atypical epithelial ells and pecimen dequacy: current laboratory practices of participants in the college of American pathologists interlaboratory comparison program in cervicovaginal cytology. Arch Pathol Lab Med 2000; 124:203-11.

43. Rodríguez E, Reynolds J, Jenkins S, Winter S, Henry M, Nassar A. Atypical squamous cells of undetermined significance in patients with HPV positive DNA testing and correlation with disease progression by age group: an institutional experience. Int J Clin Exp Pathol 2012; 5(5):428-35.

44. Massad L, Einstein M, Huh W, Katki H, Kinney W, Schiffman M, et al. 2012 updated consensus guidelines for

\footnotetext{
Contribución de autores

Guillermo Rodríguez, https://orcid.org/0000-0002-1458-1091. Diseño, ejecución, análisis, interpretación de los resultados, redacción y revisión crítica. Laura Garcia, https://orcid.org/ 0000.0001.9424.9031. Ejecución y redacción. Andrea Beracochea, https://orcid.org/0000-0002-2617-0660. Ejecución y redacción.

Rafael Alonso, https://orcid.org/0000-0001-7414-3221. Diseño, ejecución, análisis, interpretación de los resultados,redacción y revisión crítica. Benedicta Caserta, https://orcid.org/0000-0001-8446-3616. Ejecución, análisis, interpretación de los resultados, redacción y revisión crítica. Natalia Pérez, https://orcid.org/0000-0002-4166-3578. Ejecución y redacción.

Dolores Larrosa, https://orcid.org/0000-0002-6764-1246. Ejecución, análisis, interpretación de los resultados, redacción y revisión crítica. María Vero, https://orcid.org/0000-0003-3851-1239. Ejecución y redacción.

Gonzalo Ardao, https://orcid.org/ 0000-0001-5083-6584. Ejecución y redacción. Mary Nela Santos, htps://orcid.org/0000-0002-6917-6632. Ejecución y redacción.

Mary Luz Hernández, https://orcid.org/0000-0001-9607-9953. Ejecución, análisis, interpretación de los resultados, redacción y revisión crítica. Maribel Almonte, https://orcid.org/0000-0003-1623-8323. Diseño, ejecución, análisis, interpretación de los resultados, redacción y revisión crítica. Rolando Herrero, https://orcid.org/0000-0001-5448-3456. Diseño, ejecución, análisis, interpretación de los resultados, redacción y revisión crítica.
} 
the management of abnormal cervical cancer screening tests and cancer precursors. J Low Genit Tract Dis 2013; 17:S1-S27.

45. García López JA, Vilaplana Ortego E, López Segura MD, Meisels A, do Vilaplana Vilaplana E. ASCUS en citología cervicovaginal de rastreo y captura híbrida II: ¿Una quimera en nuestro medio? Rev Esp Patol 2003; 36(1):59-64.

46. Tota J, Bentley J, Blake S, Coutlée F, Duggan M, Ferency A, et al. Introduction of HPV testing as in cervical cancer screening in Canada. (Evidence Review and Report, 9 december 2015). Disponible en: http:/healthydebate.ca/wp-content/uploads/2016/04/Report-on-HPV-primary-screening.pdf [Consulta: 24 marzo 2019].

47. Herrero R, Ferreccio C, Salmerón J, Almonte M, Sánchez G, Lazcano-Ponce E, et al. New approaches to cervical cancer screening in Latin America and the Caribbean. Vaccine 2008; 26(Suppl. 11):L49-58.

48. Koliopoulos G, Nyaga V, Santesso N, Bryant A, Martin-Hirsch P, Mustafa R, et al. Cytology versus HPV testing for cervical cancer screening in the general population. Cochrane Database Syst Rev 2017; 8:CD008587.
49. Arbyn M, Ronco G, Anttila A, Meijer C, Chris J, Poljak $\mathbf{M}$, et al. Evidence regarding human papillomavirus testing in secondary prevention of cervical cancer. Vaccine 2012, 30(Suppl.5):F88-99.

50. Ronco G, Dillner J, Elfstrom M, Tunesi S, Snijders P, Arbyn M, et al. Efficacy of HPV-based screening for prevention of invasive cervical cancer: follow-up of four European randomized controlled trials. Lancet 2014; 383(9916):524-32.

51. Dillner J, Rebolj M, Birembaut P, Petri K, Szarewski A, Munk C, et al. Long term predictive values of cytology and human papillomavirus testing in cervical cancer screening: joint European cohort study. BMJ 2008; 377:a1754.

52. Anttila A, Kotaniemi-Talonen L, Leinonen M, Hakama M, Laurila P, Tarkkanen J, et al. Rate of cervical cancer, severe intraepithelial neoplasia, and adenocarcinoma in situ in primary HPV DNA screening with cytology triage: randomised study within organized screening programme. BMJ 2010; 340:c1804. 